Jaurnal of Environmental Science, Computer Science and Engineering \& Technology

An International Peer Review E-3 Journal of Sciences and Technology

\author{
Available online at www.jecet.org
}

Section B: Computer Science

Research Article

\title{
Machine Reading Comprehension: Methods and Trends of Low Resource Languages
}

\author{
Dr. Mubarak Alkhatnai \\ Department of English Language and Translation, College of Languages and Translation \\ King Saud University, Riyadh, Saudi Arabia
}

Received: 26 March 2021; Revised: 04 April 2021; Accepted: 16 April 2021

\begin{abstract}
Natural language processing (NLP) has been used to establish human-like communication with computers. Machine Reading Comprehension (MRC) has made a significant development; it has been crucial for machines to comprehend substantial language facets, like semantics, syntax, pragmatics, and phonology. Multiple studies have reported MRC models in high-resource languages. However, these models are unable to provide significant performance in MRC models in low resource languages. This is due to the unavailability of large-scale training datasets in low resource languages. Several studies on Machine Reading Comprehension (MRC) have proposed MRC models based on English. Nonetheless, these models provide insignificant performance on low-resource languages. However, limited research has been done using low resource languages, particularly Arabic, Urdu, and Hindi. This study presents a survey on trends and methods of Machine Reading Comprehension (MRC) in these low-resource languages. The survey demonstrates that available MRC models are ineffective in low resource languages, such as Hindi, Arabic, and Urdu, mainly due to large datasets and language structure unavailability. Finally, the study describes open issues in available MRC systems and provides direction for future research.
\end{abstract}

Key Words: machine reading comprehension, low resource language, question answering, trends 


\section{INTRODUCTION}

An enormous impact on humanity would emerge if computers could understand what they command to read. Machines help us in multiple ways: summarise our information, provide answers to our queries, and bring new ideas for us. There are growing appeals for the Machine Reading Comprehension (MRC) field, which is now being spun out into commercial applications ${ }^{[1-3]}$. However, there is a deficiency of such applications in low resource languages. Substantial work on popular languages like English and other languages has administered great success ${ }^{[4,5]}$. Most of the human languages, mainly low-resource language, are in dreadful need of tools and resources. These low-resource languages like Urdu, Hindi, and Arabic lack large-scale corpora, and resources are insufficient to develop statistical applications of Natural Language Processing (NLP).

There are more than 100 million speakers in the Urdu language across the world. However, it is a low resource language in the Natural Language Processing (NLP) domain in the view of NLP tools' inaccessibility and scarcity of labeled datasets ${ }^{[6,7]}$. Even though the Hindi language has more than 340 million speakers in multiple countries ${ }^{[8]}$, it is considered a low resource language. Likewise, Arabic is one of the worldwide spoken languages in Gulf areas having more than 200 million speakers. It is also a low-resource language in the Natural Language Processing (NLP) domain from NLP tools' perspective inaccessibility and limited labeled datasets ${ }^{[9]}$. The speakers of these languages are substantial, and a significant number of people are unable to speak the English Language and prefer to talk in their indigenous language. Thus, these languages lack Natural Language Processing tools, particularly for machine-reading comprehension tasks, and require more advancement in this field. Machine Reading Comprehension (MRC) is a popular way to find out the machine reading ability. Machine Reading Comprehension is an AI-complete practice that needs a Q\&A system, and the system first processes the given text, understands it, and then extracts the answer to the users' question ${ }^{[6-10]}$.

Furthermore, the machine processes the span of text and transects the answer to mimic human abilities tremendously. Consequently, machines' ability has gained enormous attention by incorporating various deep learning techniques over the past few years ${ }^{[11]}$. Nevertheless, the emerging issue with MRC is the unavailability of a large-scale corpus in multiple languages, mainly in low resource languages. Though Machine Reading Comprehension (MRC) has made various contributions in multiple ways, English reading comprehension is under its significant work dealings ${ }^{[12] .}$ It mostly dealt with rich resource languages like English due to the availability of large-scale corpora. However, current models trained on small parallel data tend to produce low-quality results, as most of the existing pre-trained models are available for only English. This challenge is exacerbated within social media. We need to enable communication using languages with no corresponding model to comprehend passages written in low resource languages such as Urdu, Arabic, and Hindi ${ }^{[13]}$.

Reading comprehension in low resource language has not been well-practiced due to large-scale corpora ${ }^{[14]}$. To the best of our knowledge, the machine reading comprehension task has not been studied yet for the Urdu Language. While in Arabic/Hindi, little work has been proposed using the BERT multi-lingual model ${ }^{[15]}$. This study critically reviews the answers offered by current research and suggests a higher bar to promote machine learning comprehension application, especially for lowresource languages. The rest of the paper is organized as follows: section 2 sheds light on the importance of low resource languages. Similarly, section 3 demonstrates challenges in machine reading comprehension. Section 4 provides a detailed summary of datasets, and section 5 discusses MRC techniques for Hindi, Arabic, and Urdu. Section 6 explains machine learning methods, and section 7 discusses deep learning techniques for low resource languages. Finally, section 8 provides discussion and analysis, and section 9 concludes the paper. 


\section{MATERIALS AND METHODS}

2.1. Importance of Low Resource Languages: A recent study deeply examined the importance of low resource languages in natural language processing ${ }^{[16,22]}$. There are around 7,000 languages globally; however, only a small fraction of these languages (20 languages) are considered highresource languages. Most significantly, low-resource languages are in dire need of NLP tools and resources to deliver benefits on a large scale. The study reported that ${ }^{[16]}$ among 7,000 languages, approximately 3000 languages do not have a writing system, and some are becoming extinct languages. It is also important to mention that half of the world's languages will be extinct by the end of this century ${ }^{[16]}$. Thus, it is essential to establish and advance the current NLP techniques to low resource languages to preserve them.

There are substantial benefits of data resources because data helps us to recognize and highlight crucial problems. Some unlabeled data sources are vital for low-resource languages. These sources include parallel data, bilingual linguistic resources, or clues from related languages. However, creating sources for low resource languages, such as labeled data, is an expensive task in terms of time and effort. It is crucial to improve the performance of available NLP tools for low resource languages. Thus, building NLP tools for such languages can shore up the association between countries and establish diversity.

Figure 1 highlights the significance of low resource languages:

1. Preservation: Upholding languages that do not have a writing system and keep the record of such languages is essential before they die out.

2. Educational applications: The advancement in NLP tools can be fruitful for educational objectives. For example, the revival of Hebrew or Gaelic made a significant improvement in NLP tools for these languages ${ }^{[16]}$.

3. Knowledge expansion: New NLP tools can help obtain fruitful and meaningful data insights — not only with statistical methods but comparing diverse languages.

4. Monitoring demographic and political processes: Some low resource languages have populations in the millions. However, they are concealed. Hence, the development of such tools can help get to monitor demographics.

Emergency response: New NLP tools can be significant to avoid catastrophic circumstances

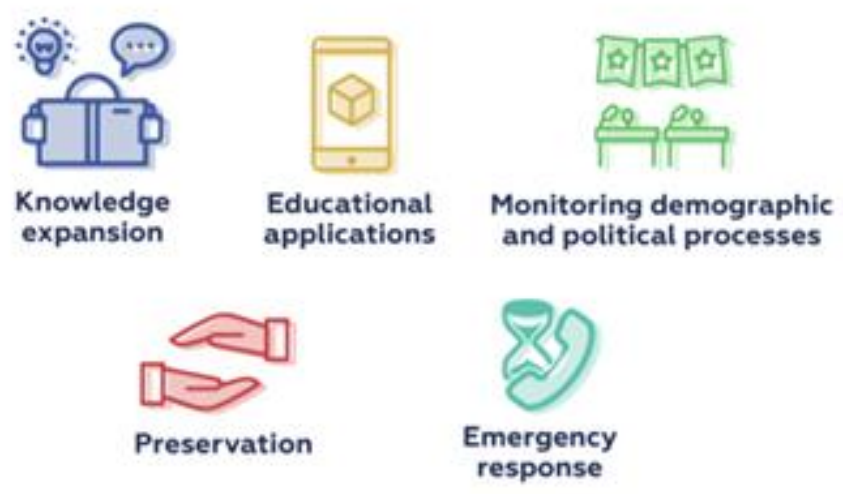

Figure 1: Significance of low resource languages 
2.2. Challenges in Machine Reading Comprehension Systems: A challenging problem in this domain is the available MRC system can only answer if the answer is present in training data used for system training. This is typically a complex problem because the system's ability to infer facts by looking at different sections of the text document, understand structured embedded content like Tables, and prepare a response to the user's query is exceptionally crucial.

Several works have shown that recent advancement in natural language processing (NLP) technology has matched or even surpassed human performance on benchmark datasets against rich languages, such as English and Chinese. Furthermore, in recent years we have seen a flurry of new datasets in rich resource languages. Each dataset has been designed to make the task challenging that hinges on implicit trivial common-sense facts.

The Challenges in Machine Reading Comprehension Systems are as follows:

1. Learn and read: Machines are required to learn the structure and meaning of language initially. Unlike humans, a machine needs to learn hidden patterns in a language and respond to the user's query, which is exceptionally crucial.

2. Understand the context: For machines, understanding the query's context, what words mesh together in a sentence is significant to formulate a reasonable response to the user query.

3. Finding the answer is not a trivial task: Like humans, machines have to investigate different parts in a passage for the right answer to the question. For example, finding an answer in a text might be challenging for a human since processing different passages requires a long time. However, this becomes crucial for machines since the answer might be in other sentences of the passage, and the query requires context-based answers.

2.3. Low resource languages' dataset summary: The table given below gives a summary of MRC techniques for Hindi, Arabic, and Urdu language. From the table, we can see an abundance of research papers and work found on Arabic and Hindi Languages when it comes to datasets. However, only one article on the Urdu language could be found, which did not contain any evaluation dataset. So, we could not perform a more in-depth analysis of the Urdu language. Table 1 shows a whole outline of the techniques multiple papers used and the limitations we observed.

Table 1: MRC techniques for Hindi, Arabic, and Urdu

\begin{tabular}{|c|l|l|l|l|l|l|}
\hline $\begin{array}{l}\text { Model } \\
\text { Performance }\end{array}$ & Arabic & $\begin{array}{l}\text { Not } \\
\text { reported }\end{array}$ & $\begin{array}{l}\text { Information } \\
\text { Retrieval with NLP } \\
\text { techniques }\end{array}$ & 2002 & $\begin{array}{l}\text { Tataset } \\
\text { Only known categories } \\
\text { of question, Limited } \\
\text { dataset }\end{array}$ & Not reported \\
\hline$[17]$ & Arabic & $\begin{array}{l}\text { Not } \\
\text { reported }\end{array}$ & $\begin{array}{l}\text { Information } \\
\text { Retrieval }\end{array}$ & 2012 & $\begin{array}{l}\text { Only factoid questions, } \\
\text { few words answer }\end{array}$ & Accuracy=19\% \\
\hline$[18]$ & Arabic & $\begin{array}{l}\text { Quranic } \\
\text { Corpus } \\
\text { Ontology } \\
\text { and Qurany } \\
\text { Ontology }\end{array}$ & $\begin{array}{l}\text { Preprocessing, } \\
\text { classification, and } \\
\text { Information } \\
\text { Retrieval }\end{array}$ & 2014 & $\begin{array}{l}\text { Limited to questions } \\
\text { related to the Holy } \\
\text { Quran, a pre-listed } \\
\text { concept only }\end{array}$ & Precision=0.73 \\
\hline & & &
\end{tabular}




\begin{tabular}{|c|c|c|c|c|c|c|}
\hline [20] & Arabic & $\begin{array}{l}\text { Not } \\
\text { reported }\end{array}$ & $\begin{array}{l}\text { Semantic analysis } \\
\text { and Standford } \\
\text { parser }\end{array}$ & 2014 & $\begin{array}{l}\text { Logical transformation, } \\
\text { questions must be in the } \\
\text { same passage }\end{array}$ & Precision $=83.3 \%$ \\
\hline [21] & Arabic & $\begin{array}{l}\text { Not } \\
\text { reported }\end{array}$ & $\begin{array}{l}\text { Information } \\
\text { Retrieval }\end{array}$ & 2016 & $\begin{array}{l}\text { Only addressed 'Ws' } \\
\text { question type, rule-based }\end{array}$ & Accuracy $=78 \%$ \\
\hline [22] & Hindi & $\begin{array}{l}\text { MMQA in } \\
\text { Hindi }\end{array}$ & $\begin{array}{l}\text { CNN, GRU, and } \\
\text { RNN based Multi- } \\
\text { domain Multi- } \\
\text { lingual QA } \\
\text { framework }\end{array}$ & 2018 & $\begin{array}{l}\text { Translation, Semantic } \\
\text { differences, few words } \\
\text { answer, limited dataset }\end{array}$ & $\begin{array}{l}\text { Exact Match=30.24\% } \\
\text { on Factoid } \\
\text { Exact Match=40.19 } \\
\text { on Descriptive }\end{array}$ \\
\hline [23] & Urdu & $\begin{array}{l}\text { Not } \\
\text { reported }\end{array}$ & $\begin{array}{l}\text { Levenshtein } \\
\text { distance, POS tag }\end{array}$ & 2019 & $\begin{array}{l}\text { Only for Roman Urdu, } \\
\text { only limited Roman } \\
\text { structures }\end{array}$ & $\begin{array}{l}\text { Not specified any } \\
\text { matrix }\end{array}$ \\
\hline [24] & Arabic & $\begin{array}{c}\text { cQA } \\
\text { dataset }\end{array}$ & SVM & 2019 & $\begin{array}{l}\text { Slow, single answers, no } \\
\text { dependency } \\
\text { consideration }\end{array}$ & $\mathrm{MAP}=42.20$ \\
\hline [25] & Hindi & $\begin{array}{l}\text { standard } \\
\text { Hindi, } \\
\text { SQuAD }\end{array}$ & $\begin{array}{l}\text { Neural network for } \\
\text { Multi-lingual }\end{array}$ & 2019 & $\begin{array}{l}\text { Factoid questions, } \\
\text { Translation to Hindi, } \\
\text { worked only ion } \\
\text { Translated SQuAD }\end{array}$ & $\begin{array}{l}F 1=44.97 \text { on Hindi } \\
\text { Specific } \\
F 1=53.77 \text { SQuAD }\end{array}$ \\
\hline [26] & Hindi & $\begin{array}{l}\text { Facebook } \\
\text { research }\end{array}$ & LSTM and GRU & 2019 & $\begin{array}{l}\text { Code-mixed languages, } \\
\text { labeled answers, pre } \\
\text { summarisation of context }\end{array}$ & $\begin{array}{l}\text { Accuracy }=97.3 \text { on } \\
\text { LSTM } \\
\text { Accuracy }=98.9 \text { on } \\
\text { GRU }\end{array}$ \\
\hline [27] & Hindi & $\begin{array}{l}\text { The }(20) \\
\text { QA bABl }\end{array}$ & $\begin{array}{l}\text { Memory network, } \\
\text { RNN, LSTM, and } \\
\text { GRU }\end{array}$ & 2019 & $\begin{array}{l}\text { Code-mixed Hindi } \\
\text { Language, slow, small } \\
\text { dataset }\end{array}$ & $\begin{array}{l}\text { LSTM } \\
\text { Accuracy }=66.3 \\
\text { GRU accuracy=81 } \\
\text { RNN accuracy=65 }\end{array}$ \\
\hline [28] & Arabic & $\begin{array}{l}\text { ARCD, } \\
\text { Arabic- } \\
\text { SQuAD }\end{array}$ & $\begin{array}{l}\text { Hierarchical TF- } \\
\text { IDF, BERT }\end{array}$ & 2019 & $\begin{array}{l}\text { Semantic meaning not } \\
\text { considered, } \\
\text { inconsistency in answer }\end{array}$ & $\begin{array}{l}\text { F1 }=51.3 \text { on } A R C D \\
F 1=48.6 \text { on Arabic- } \\
\text { QuAD }\end{array}$ \\
\hline [29] & $\begin{array}{l}\text { Hindi, } \\
\text { Arabic }\end{array}$ & $\begin{array}{l}\text { Translated } \\
\text { SQuAD }\end{array}$ & $\begin{array}{l}\text { Multi-lingual } \\
\text { BERT, Cross- } \\
\text { lingual transfer }\end{array}$ & 2019 & $\begin{array}{l}\text { Dataset, translation, very } \\
\text { brief answer }\end{array}$ & Exact Match=59.6 \\
\hline [30] & Hindi & $\begin{array}{l}\text { XQuAD, } \\
\text { SQuAD }\end{array}$ & $\begin{array}{l}\text { Multi-lingual } \\
\text { BERT }\end{array}$ & 2020 & $\begin{array}{l}\text { The semantic difference } \\
\text { in translation, passage } \\
\text { level indexing, do not } \\
\text { address the descriptive } \\
\text { question }\end{array}$ & $\begin{array}{l}F 1=78.83 \text { on } X Q u A D \\
F 1=88.76 \text { on SQuAD }\end{array}$ \\
\hline
\end{tabular}

Furthermore, we also studied different datasets for Hindi, Arabic, and Urdu languages. The datasets were extracted from several sources such as Wikipedia, Factoid articles, stories, cQA, Holy Quran, 
and Facebook. Additionally, it is observed that when it comes to NLP research for a low-resource language like Urdu, there is an insufficient amount of data present. In Table 2, we have given the Low resource languages dataset summary.

Table 2: Low resource Language's dataset summary

\begin{tabular}{|c|c|c|c|c|c|}
\hline Dataset & Language & Type & Size Generation & Corpus & Source \\
\hline $\begin{array}{l}\text { Translated } \\
\text { SQuAD }\end{array}$ & Hindi & $\begin{array}{l}\text { Extractive } \\
\text {,Natural }\end{array}$ & $\begin{array}{l}\text { 18454Question-passage-Answer } \\
\text { triplets }\end{array}$ & Crowd-sourcing & Wikipedia \\
\hline $\begin{array}{l}\text { Translated- } \\
\text { XQuAD, } \\
\text { SQuAD } \\
{ }^{[30]}\end{array}$ & Hindi & Extractive & $\begin{array}{l}240 \text { parar with } 1190 \mathrm{QA}, 100 \mathrm{k}+ \\
\text { questions }\end{array}$ & Crowd-sourcing & Wikipedia \\
\hline $\begin{array}{l}\text { MMQA } \\
\text { [31] }\end{array}$ & Hindi & $\begin{array}{l}\text { Factoid, Short } \\
\text { Descriptive }\end{array}$ & $\begin{array}{l}500 \text { articles against } 250 \text { languages } \\
\text { and have } 5.4 \mathrm{k} \text { question-answer } \\
\text { pairs }\end{array}$ & Self-created & $\begin{array}{l}\text { Factoid } \\
\text { articles }\end{array}$ \\
\hline $\begin{array}{l}\text { Facebook } \\
\text { research }^{[26]}\end{array}$ & Hindi & Extractive & contained $11 \mathrm{k}$ stories & Not disclosed & Stories \\
\hline $\begin{array}{l}\text { Translated } \\
\text { SQuAD }^{[29]}\end{array}$ & $\begin{array}{l}\text { Hindi, } \\
\text { Arabic }\end{array}$ & Extractive & $\begin{array}{l}\text { 12k sentences in English and } 6 \mathrm{k} \text { in } \\
\text { Hindi and Arabic }\end{array}$ & Crowd-sourcing & Wikipedia \\
\hline $\begin{array}{l}\text { ARCD, Arabic- } \\
\text { SQuAD }\end{array}$ & Arabic & Extractive & $\begin{array}{l}155 \text { articles } 250 \text { characters each, } \\
536 \text { articles having } 107 \mathrm{k} \text { paragraphs }\end{array}$ & $\begin{array}{l}\text { Crowd- } \\
\text { sourcing }\end{array}$ & Wikipedia \\
\hline cQA dataset ${ }^{[24]}$ & Arabic & Extractive & $23 \mathrm{M}$ words & $\begin{array}{l}\text { Crowd- } \\
\text { sourcing }\end{array}$ & cQA website \\
\hline $\begin{array}{l}\text { Quranic Corpus } \\
\text { Ontology }{ }^{[31]}\end{array}$ & Arabic & Extractive & 6236 verses and 1217 concepts & Self-created & $\begin{array}{l}\text { The Holy } \\
\text { Quran }\end{array}$ \\
\hline QA bAB1 ${ }^{[27]}$ & Hindi & Extractive & 10k examples as stories & Not specified & $\begin{array}{l}\text { Facebook } \\
\text { Research }\end{array}$ \\
\hline
\end{tabular}

2.4. Challenges in Low Resource Languages: Most of the research in this field aims to solve data problems. Moreover, reading comprehension is strenuous, and therefore machines can be taught by providing a substantial amount of data to address this issue. This study's primary purpose is to present a survey on Machine Reading Comprehension methods, particularly on low resource language. The first thing that we noticed is that there is a lack of data, which hinders research. Due to the limited data, the amount of study that could be done was also limited. Another challenge associated with semantics is that there are substantial language models where syntax is considered during translation. The current MRC models were incapable of this function. Table 3 gives a brief rundown of critical problems and challenges in low resource languages. 
Table 3: Challenges in Low Resource Languages

\begin{tabular}{|c|c|c|}
\hline Challenge & Description & Reference \\
\hline $\begin{array}{l}\text { Multiple writing } \\
\text { styles }\end{array}$ & $\begin{array}{l}\text { There are multiple writing styles in low-resource } \\
\text { languages. Particularly, Urdu and Hindi have a Roman } \\
\text { writing style. In Roman writing, no grammar or formal } \\
\text { rules are followed, and one word can be written in } \\
\text { multiple ways. For example, the term "Coronavirus" can } \\
\text { also be written as "Koronawirs", "koronavirus" or } \\
\text { "coronavirus" or "coronavirus" in Roman Urdu. Thus, to } \\
\text { find the possible ways a word can be written, this requires } \\
\text { text from a bigger audience. }\end{array}$ & [23] \\
\hline Data scarcity & $\begin{array}{l}\text { Language models, especially deep learning models, such } \\
\text { as neural networks and transformers-based models, } \\
\text { require substantial annotated data for model training. } \\
\text { However, the limited annotated dataset is available } \\
\text { because data annotation requires financial sources and } \\
\text { time. }\end{array}$ & [25] \\
\hline $\begin{array}{l}\text { Lack of } \\
\text { capitalisation }\end{array}$ & $\begin{array}{l}\text { As compared with English, low-resource languages do not } \\
\text { have capitalization. This makes it challenging to identify } \\
\text { proper nouns, abbreviations, and acronyms. For example, } \\
\text { Urdu does not have an upper case, and therefore this poses } \\
\text { challenges in various tasks to determine the proper noun. }\end{array}$ & [25] \\
\hline $\begin{array}{l}\text { Semantic } \\
\text { challenges }\end{array}$ & $\begin{array}{l}\text { Semantic problems in multi-lingual modeling where the } \\
\text { only syntax is considered during translation. So, the } \\
\text { translation of datasets from rich-resource languages to } \\
\text { low-resource languages induces semantic issues due to the } \\
\text { difference in grammatical structure, and eventually, the } \\
\text { word meanings are changed. }\end{array}$ & [28] \\
\hline $\begin{array}{l}\text { Insufficient } \\
\text { benchmark } \\
\text { datasets }\end{array}$ & $\begin{array}{l}\text { There is a scarcity of benchmark datasets in different tasks } \\
\text { to evaluate machine learning models' performance, mainly } \\
\text { in automatic machine reading comprehension. Especially, } \\
\text { standard benchmark datasets are insufficient to evaluate } \\
\text { and compare various proposed techniques. }\end{array}$ & [29] \\
\hline Scalability issues & $\begin{array}{l}\text { Low-resource languages also have scalability issues due } \\
\text { to the different knowledge domains in rich-resource } \\
\text { languages. For example, suppose the task is to develop an } \\
\text { Arabic language system related to "Islamic hadith". In that } \\
\text { case, however, the proposed model trained through multi- } \\
\text { lingual modeling cannot perform to hadith due to the } \\
\text { invalidity of datasets related to hadith in English. }\end{array}$ & [29] \\
\hline $\begin{array}{l}\text { Anaphora } \\
\text { structures and }\end{array}$ & $\begin{array}{l}\text { Different kinds of ellipsis and anaphora structures } \\
\text { compared to English or other rich languages due to }\end{array}$ & [30] \\
\hline
\end{tabular}




\begin{tabular}{|l|l|c|}
\hline Ellipsis & $\begin{array}{l}\text { grammatical differences. Different structures can lead to } \\
\text { different semantic meanings. }\end{array}$ & [31] \\
\hline $\begin{array}{l}\text { Limited } \\
\text { knowledge base }\end{array}$ & $\begin{array}{l}\text { Low-resource languages contain insufficient knowledge } \\
\text { bases such as Wikipedia. Therefore, datasets are } \\
\text { inadequate due to the limited knowledge base. }\end{array}$ & \\
\hline
\end{tabular}

\section{MRC in Machine Learning}

The world of Natural Language Processing (NLP) is rather extensive and comprehensive, which leaves a lot of room for improvement. Standard NLP models require large amounts of training data and/or sophisticated language-specific engineering. We know that NLP is mainly data-driven; a question that needs utmost attention is what kind of data and how much data needs to be used. Due to such a large amount of training data set, even if the language selected is spoken by millions of people, like the whole Bantu family, it is almost impossible to find a linguistically trained speaker to build a language model for different languages. Therefore, we have to find a way to implement NLP in lowresource settings.

As deduced by the research, there are two main approaches to NLP in low-resource settings. In both of these approaches, the amount of data and knowledge regarding the selected language are rendered insufficient. The first approach is a traditional approach that mainly focuses on collecting data for a single or a variety of languages. In this approach, we start with a data collection phase for lowresources languages such as Hindi, Urdu, Arabic, etc. The data collected needs to be done by an expert. However, due to each language being unique, we have to devise a data collection strategy from scratch. The other approach is to apply transfer learning, which is where machine learning comes into play. Transfer learning is a research problem that focuses on storing data and knowledge gained when searching for one issue and applying the acquired knowledge to a different but related problem. For example, suppose that you gain some knowledge while learning about cars. Now theoretically, you could apply that knowledge when trying to recognize trucks. Similarly, you could use transfer knowledge when learning about a specific language, i.e., Hindi, and using the gained knowledge to identify another language like Urdu or Arabic. As deduced above, machine learning has emerged as a revolutionary subject for text reading and extracting concepts. Linguists have been studying it as an obsession for the past few years to implement it, which motivated us to do the same

Our end goal is to study a language, apply appropriate machine learning algorithms, train a model, and use the trained model to predict words correctly. We have termed this as a binary classification problem, where we use supervised learning to predefined categories. By doing so, we can transform any document and query pair into a set of new "document-query" pairs so that a certain subset corresponds to a specific entity within a particular way. For example, if you want to guess an entity that fits a blank, there are two subsets. One subset correctly responds to the blank, and the other one reacts negatively. To top it off, we have also associated some features with the pairs, as feeding the entire pair into a machine learning model is infeasible at this point.

3.1. MRC in the Arabic language: The machine learning-based approach for MRC in Arabic is different from the rule-based MRC approach, as the previous approach was only restricted to a few words. We had to conduct an extensive keyword matching with no contextual consideration and elaboration in the last approach, presenting many challenges. To tackle multi-sentence question answers with a courtesy fragment, and lexical and semantic dependencies consideration and different 
Arabic QA scheme model ${ }^{[24]}$ was proposed. The research was based on a support vector machine (SVM) with advanced text representations achieved through tree kernels. Tree kernels are very helpful in processing natural language as they help us study classification and provide ways to improve traditional methods. The tree kernels form a parse tree, which figures out the textual similarities and word embedding. Regarding the candidate questions' problem, which was also faced in the rule-based MRC model, machine learning-based MRC takes a different approach. In this approach, heuristic retrieval is employed to obtain the candidate questions, ranked based on the candidate question-answer extraction pair. The final answer is then selected from this extraction pair.

3.1.1. QA system for the Holy Quran called Al-Bayan: The previous QA system ${ }^{[24]}$ was limited to tackling basic multi-sentence questions. A highly accurate QA system ${ }^{[31]}$ was proposed to address general to domain-specific questions. The QA system, called Al-Bayan, was explicitly designed for the Holy Quran, and it was based on three primary modules. The first module focuses on question analysis, which includes pre-processing and classification of information. In the second module, the information retrieval process is applied to the verse containing the required answer. Moreover, finally, entity recognition and feature extraction are performed. All in all, 6236 verses of the Holy Quran were classified into 1217 concepts by taking Arabic questions as input and returning the relevant verse as an output.

3.2. MRC in Deep Learning: Another area of research that grabbed our attention is deep learning and the power of neural networks. A problem with machines is that, unlike the human mind, they cannot read a full sentence and deduce what the sentence means on its own. Instead, these machines only learn a few heuristics about the text and then make educated guesses based on these heuristics. A human mind is advanced enough to understand the underlying meaning of a sentence and make meaningful inferences based on their understanding of the sentence.

To address this problem, we decided to make use of the concept of deep learning and incorporate the power of neural networks onto our system. Deep learning is a phenomenon that enables machines and tools to mimic the human brain and process the data given to it. After processing the input data, machines can detect objects, recognize speech, translate languages, and make decisions independently without human supervision. Deep learning incorporates neural networks within its architecture. Neural networks have become a hot development in machine learning, as it consists of several algorithms that allow us to learn more complex functions. Just like the human brain uncovers patterns and connections in a dataset, neural networks enable machines to do that same, which is perfect for the kind of system we want to develop.

Deep learning comes in very handy when dealing with word embedding or word vectors as they are the cornerstone of many NLP approaches. Word embedding is a learned representation for a particular text where words with the same meaning can be represented the same way. It is a technique where each word is assigned a real-valued vector in a predefined vector space. An individual word is mapped against a single vector, and the values are learned in a way that resembles a neural network. The phenomenon is considered a breakthrough in deep learning as it directly addresses NLP's problems.

3.3. MRC in the Arabic language: Despite many people who speak and use Arabic daily (more than 200 people) and many countries (more than 30), which consider Arabic as their first official language, there is minimal research to use deep learning for Arabic queries. Compared to the English Language, Arabic has an entirely different orthography that goes from right to left. Due to its complexity, we have to work with only a few MRC papers that we found. The first thing that we noticed is that all the previous approaches which tried to mix MRC and Arabic were all rule-based. However, the maximum 
number of rules was only confined to 14, which is insufficient for MRC purposes. Out of all the work that has been done in the past, the one that stood out was proposed by presented a rule-based QA approach for Arabic questioning and answering ${ }^{[21]}$. He devised the system consisting of three significant modules involving question analysis and understanding, passage retrieval, and the answer extraction and response generation module.

In the first module, the most crucial step is carried out, which question is understanding. In common languages like English, regardless of the type of question being asked, every question falls in the category of who, what, why, when, where, and how $(5 \mathrm{Ws}$ and $1 \mathrm{H})$. However, that is not the case with Arabic, as there is more than one representation of interrogatives. So, 95+ rules were defined for a detailed question analysis. After conducting the research, the questions and context were translated into the English Language for text processing. The text was segmented into tiny parts, and a semantic tree was built. After a semantic tree was constructed on the Stanford parser's base, the text was translated from English to Arabic. Finally, the candidate answers were extracted and ranked based on the allocated score.

3.4. Arabic MRC Question Answering Approach; Now, we move on to the next problem. A lot of times, reading comprehension tasks tends to get quite tricky. For example, we might be required to turn our focus on "fill in the blank" style questions or multiple-choice questions. These queries are different from the ones discussed above, as the model cannot focus on a particular part to find the answer to the query. While it may sound simple for a human to choose the right choice that fits the blank, it is not that simple for machines to do so.

To address the problem related to multiple-choice questions and answer tasks from a piece of text, an Arabic MRC ${ }^{[18]}$ question answering approach based on Information Retrieval (IR) was introduced. The approach presented a shallow process that was developed for Arabic test understanding. Whenever the system needed to infer text, it used IR methods to conclude. In such an NLP system, anaphora is a common challenge that needs to be addressed. Anaphora frequently rises within texts and oral discourse, so we need to figure out the corresponding referent's antecedent in Anaphora Resolution. After the problem is figured out, a four-step mechanism is introduced to build the shallow method. It begins with a question analysis where all the stops in the question are removed, and the remaining words are saved as question focus. The second step involves aligning the passages with the items for respective questions. In the end, the final answer is given by selecting the right passage from the data collected. In case no solution could be deduced, a list of reference pairs of words is included from the background collection. Figure 2 shows the architecture of the model.

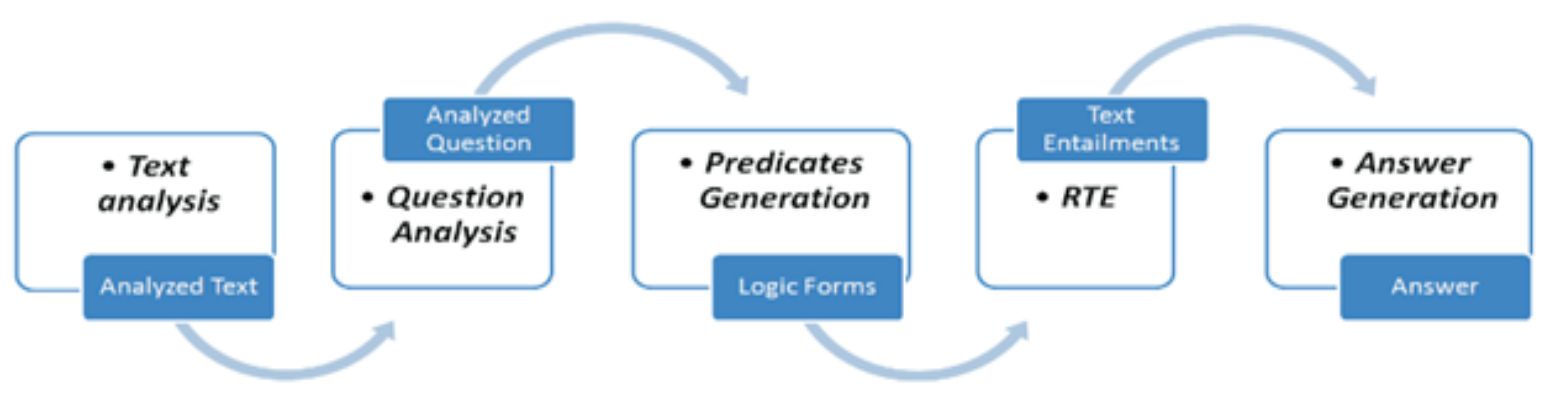

Figure 2: Text and question are analyzed and passed to the logic block for text entailment to generate a candidate answer ${ }^{[18] .}$ 
3.5. Attention-based: This paper aims to find out the best possible way to train datasets by comparing all available methods. So, the next question that we face is, can we do better than transfer learning when it comes to NLP? Well, the answer to this problem is a clear and straightforward yes. When we reach such a high level of programming, we want to make sure that our model thinks and works just like a human brain does. For example, when we, humans, perform a comprehension task that involves answering some questions, we do not read the whole text and then look at queries to finalize the answer to those queries. Instead, we look at the queries for clues about which words we should pay attention to in the text. So, at the most basic level, we are only concentrating on relevant things to find within a whole text.

3.6. MRC in the Hindi Language: All of the proposed approaches in this paper until now take the language structure into account for computing MRC tasks. Now that we have looked at attentionbased strategies for rich language and how documents can be read and answered, let us divert our attention to using deep neural networks for multi-lingual QA tasks in the Hindi language. Some major work ${ }^{[25]}$ proposed a deep neural network for multi-lingual tasks, including the Language Hindi. He used this system to make a model in which the model's input consisted of two things: a factoid question and a contextual snippet. An output snippet was extracted from the given contextual passage purely based on an attention-based approach by processing these two together. The model exploited the lexical and semantic commonalities between two or more sentences using a language-independent graph-based algorithm. The SQuAD dataset, translated into the Hindi language, was used to propose a network model. The diagram below shows the proposed deep neural network for multi-lingual QA.

3.6.1. SQuAD Dataset for MRC in Hindi: The SQuad or the Stanford Question Answering Dataset is a reading comprehension dataset consisting of crowd workers' questions on Wikipedia articles. The answer to every question contains a segment of text from its corresponding passage, or the problem may be unanswerable. Having a considerable resource of 100,000+ question-answer pairs for more than 500 articles, this dataset is comprehensively larger than any other dataset that we have previously used.

Furthermore, SQuAD v1.1 is specially designed for MRC, which is used for a system that could read a document. After analyzing the paper, the system can give relevant answers to queries, just like a human would. SQuAD v2.0 was also developed to assist in reading comprehension for systems to conclude a question or query.

3.7. Multi-lingual QA Framework for English and Hindi A QA system aims to develop a model to automatically generate the answer for a given question from the data repository. Posing questions in different languages and retrieving the answers to these questions accordingly is known as multilingual question answering. For example, the question's language may differ from the language of passage where the answer lies. This created a problem for our previously developed model, which is why it needs to be addressed. To address this problem, we study a research ${ }^{[22]}$ that devised a system to handle a multi-domain and multi-lingual QA framework for English and Hindi languaFor the dataset, he gathered 500 articles from the web, having 250 articles for each language. There were a total number of $5.4 \mathrm{k}$ question-answer pairs, which were created for each language. The purpose of this research was to ask questions in one language and get their answers in another.

The system used a translation-based approach and translated the question-answers pairs from the English Language to the Hindi language to fulfill this task. After the Hindi articles and questions were translated into English, the translated text was indexed at the passage level through an inverted indexing mechanism. The translated items were then classified using CNN and RNN based deep learning classifiers, which provided the needed semantic constraints for the required answer. We can 
divide the proposed model into four different layers. The first layer, named the question embedded layer, was used to transform the items into a real-value dense vector as word embedding.

The second layer, called the convolutional layer, obtained the feature vector from the set. The recurrent or the third layers worked to get the forward and backward hidden states at each time step through the gated recurrent unit (GRU). And finally, the last layer is called the Softmax classification layer, which classified the resulting states. After completing the questions, query formation by removing all the stop words from the question sentence. By doing so, all the nouns, verbs, and adjectives in the sentence were concentrated so that the question could be ranked. The final correct answer was determined based on classification and ranking, which was then translated back to Hindi to establish a multi-lingual QA model. One of the model's downsides is that it was only confined to a few words and a limited dataset as we have to convert Hindi into English and then back to Hindi.

3.8. MRC Model for Code-Mixed Hindi Language: For the sequence modeling on the code mixed the Hindi language, a lot of work has been done by different researchers. First, we review a study [27] that proposed a deep learning-based MRC model for code-mixed Hindi language. Based on the GRU, LSTM, and RNN sequence models for observing the output, the end-to-end memory network found that GRU was the most suitable and accurate sequence model compared to other models. The MRC system depended on passing the controller module of the end-to-end memory network. The controller's output was further passed to the memory module along with the input sentences. Here is a model representation of how the system is handed to the LSTM and GRU layers to predict the answer.

One of the most significant benefits of using LSTM with GRU is that LSTM can retain or remember information for a more extended time than other sequence models. It can also not forget words over a comprehensive range of sentences and can even forget information whenever needed. So, LSTM can determine which information is essential for the system and which data should be disregarded. To put this theory into action, LSTM and GRU-based combined mechanisms for MRC ${ }^{[26]}$ were used on mixed Hindi data. The dataset was collected after conducting a Facebook search so that raw data could be generated. The dataset contained $11 \mathrm{k}$ stories and passages, which included a question after a certain number of lines. So, the system had to keep in mind after how many lines is a question asked. The answers were followed by the questions, which were also labeled to define a range of answer spaces. The proposed system was passed through the GRU/LSTM units for encoding and decoding and eventually passed through Softmax for answer prediction.

3.9. Zero-Shot Multi-lingual MRC QA for Different Languages: All the approaches that we have previously described worked on the same principle. These approaches assumed that the question and the context were in the same language. If the problem and the context were not in the same language, they were translated to a baseline language like English. There is a system ${ }^{[30]}$ where you had to quote an answer from a given passage. However, the answer to the question given from the passage and the original question could be from two different languages. It required taking the question from a lowresource language and taking the benefit of a rich language to extract the answer through multi-lingual modeling. The system was inspired by the multi-lingual BERT model, which contained 104 different languages except for Hindi. To overcome this problem, the study was tuned to the prior network to accommodate English and Hindi question answering simultaneously. It was done using an extended version of the XQuAD dataset to include zero-shot baseline, Hindi monolingual QA augmentation, and Hindi cross-lingual QA augmentation.

3.10. Information Retrieval and MRC: Information retrieval is the science of fetching and searching for specific information from a document and looking for metadata containing text, images, or sounds. When combining MRC with rich languages like English, traditional information retrieval 
techniques are quite common. As the model has a whole database where it can fetch information, it becomes easy to do so. However, when it comes to the Arabic language, this gets quite complicated. An Arabic MRC QA framework called QARAB ${ }^{[17]}$, worked based on combining the concepts of information retrieval with the natural language processing approach. Data were extracted from the question and candidate document, which was retrieved by the IR system. After removing all the needed data, the keyword matching strategy was coupled with structure matching to find relevant questions.

On the one hand, the IR system was used to search documents pertinent to the query. The NLP system was used to build an Arabic lexicon for tokenization, tagging, feature extraction, and identification of proper nouns. All the questions were then manually classified based on a set of known and predefined question categories.

\section{TRANSFER LEARNING}

Humans have an inherent ability to learn a specific task and transfer the gained knowledge across several other studies. The more related both these tasks are, the easier it is for us to share or crossutilize our understanding. The conventional machine learning and deep learning algorithms we have designed so far are optimized to work in isolation. By design, they are not able to transfer knowledge gained from one task onto another task. To tackle this problem, transfer learning is implemented to machine learning and deep learning algorithms so that isolated learning paradigms can be bypassed to utilize knowledge gained across several tasks.

4.1 Cross-Lingual Transfer Learning: One of NLP cornerstones is to combine language models and transfer learning so that the problem of low-resource languages having a large dataset can be solved. By using the transfer learning approach, we find certain commonalities between the two languages. By exploiting the similarities between the two languages, a standard model is built. This language model, which is made for one language, is used to develop more models. For example, we can transfer the data from resource-rich source languages to resource-poor language so that we do not have to build a new language model from scratch.

4.2 Transfer of Annotations: NLP systems rely heavily on annotated datasets. When such datasets are unavailable, we use transfer learning methods to develop a model based on transferring annotations from one or more rich-resource languages to our interest's target language. We can divide these models into two approaches: 1) where the annotation is projected from parallel data using supervised models, 2) direct model transfer from annotated datasets in rich-resource languages. However, if we want to train such models using cross-lingual bridges, we require extensive linguistic knowledge and resources about the relation between the source language and the target language.

4.3 Transfer of models: On the other hand, the transfer of models refers to training a resource-rich language and applying everything learned from this language onto a resource-poor language in zeroshot or one-shot learning. Zero-shot learning is a problem in machine learning in which a learner is trying to observe samples from classes that were not attended during the training procedure. Therefore, he needs to predict the category that the models belong to so that everything goes into the right place. Likewise, a one-shot sample is a classification method in which one or a few models are used to classify new models in the future. This technique is particularly prevalent in machine translation, where the samples collected from a rich-resource language pair are used to predict a lowresource pair.

4.4 Arabic MRC System for Open Domain Arabic QA Tasks (SOQAL): When it comes to tackling open-domain Arabic QA tasks, an Arabic MRC system was proposed ${ }^{[28]}$ called (SOQAL). 
The system extracted articles from Wikipedia for Arabic questions and removed answers by translating them with the help of SQuAD. SOQAL is a two-step system used for open-domain Arabic QA tasks, where Wikipedia is the knowledge base. The first step involved document retrieval through the Hierarchical TF-IDF mechanism. In the second step, the MRC model using a pre-trained bidirectional BERT transformer was used. The Hierarchical TF-IDF mechanism helped the reader reduce the research space as only such documents were selected, most relevant to the question. The system did not have to carry out a full-on search, which saved a lot of time. Moreover, the pre-trained BERT document reader, which was used in the system, embedded the vocabulary and questionanswer pairs to estimated log-likelihood. Finally, after the relevant questions were put into the system, and the vocab and question-answer pairs were implanted, the candidate answers obtained were ranked to answer the highest score.

Another Arabic dataset named ARCD was presented in the paper, which contained almost 1395 questions, which were also taken from Wikipedia by doing machine translation of Arabic-SQuAD. The ARCD dataset consisted of a total of 155 articles that included more than 250 characters each. Another 235 articles were translated using Google NMT translator, which contained 48344 questions from the 10354 paragraphs obtained from them. In total, roughly 269k sections had 233 characters in each of them. In the end, the answers from the ARCD dataset were manually categorized into numerical and non-numerical forms, and the questions were labeled on the word match, syntactic variation, ambiguous and multiple sentence reason, etc.

\section{BENEFITS OF MACHINE READING COMPREHENSION}

There are multiple benefits of MRC, and we shed light on some of the most crucial pros of MRC systems.

5.1. Appropriate retrieved Answers: Machine Reading Comprehension is a platform where anyone can retrieve the information they want. And this machine will be very beneficial for users in many ways. Users can even retrieve information in car manuals or dense tax code documents easily and quickly using Machine Reading Comprehension. This machine is also very beneficial for lawyers, doctors, and experts in any field ${ }^{[36]}$. They can promptly retrieve their medical findings specifically or generally without investing so much time. This fast and quick search system aids the experts to treat more patients than search for their specific results.

5.2. Chatbots for customer support: Machine Reading Comprehension displays a vast range of applications ${ }^{[37]}$. Social companies, including Google, Facebook, and Twitter, utilize this machine to extract and organize a significant input data volume. Similarly, Machine Reading Comprehension has a beneficial aspect for smaller-scale, including AdmitHub and Allganize. AdmitHub uses this machine to develop chatbots for college enrollment, and Allganize develops chatbots to improve customer service.

5.3. Social media or news media websites: As an organization (news media website) or person posts their personal opinions on social media websites like Instagram, Twitter, Facebook, etc. These opinions may be on political issues, Islamic, Economic and social issues. So it is not straightforward to find out the answer snippets for the respective statement from these social websites. People use "AND', 'OR," Filetype' etc., to resolve their queries. But now, MRC made it more accessible and comfortable for society. Someone can easily retrieve the desired data by MRC and natural language ${ }^{[38]}$. This machine can provide the same snippet quickly that users want. 


\section{OPEN ISSUES}

A literature study reveals many issues with machine reading comprehension (MRC) that remained unsolved. The following open issues create a gap between MRC applications and their users.

6.1. Limitation of Given Context: Machine reading comprehension (MRC) retrieves the answers to questions based on given data. The provided context is significant for the user and machine to work efficiently because the MRC task is based on words. But sometimes, given context restricts the device. If the given context consists of multi-passages, MRC could not perform well. It may skip some important features of given information or understand the whole context ${ }^{[32]}$. That is why the MRC task is unable to predict the exact answer. To improve machine reading comprehension efficiency, it needs to introduce the features that deeply retrieve the information.

6.2. Robustness of MRC Systems: Based on the literature analysis, most MRC models proved ineffective in overlap context ${ }^{[33]}$. The MRC model called Stanford Question Answering Dataset (SQuAD) drops MRC tasks dramatically in adversarial questions. Because in this model, a distracting sentence is added to give data that has to be answered. This distracting sentence mainly confuses the MRC practice but does not affect the answer. The drawback of this model is that it decreases the MRC performance. To overcome this flaw, the robustness of the MRC system should be elevated to face any context.

6.3. Incorporation of External Knowledge: To improve the MRC system efficiency, a knowledge base MRC system has been introduced. This model works on the principle of the human mind. As the human mind uses previous knowledge to find out any query, knowledge base MRC systems also use previous but relevant information called external knowledge to retrieve the answer. But a machine could not work as a human mind. MRC system background information is scattered in knowledge bases, and devices cannot organize the data like the human brain ${ }^{[32] .}$

Moreover, it requires additional human effort, and relevant previous knowledge could not be found directly. And to solve the query, more relevant data is needed. In short, the MRS system has to be studied more.

6.4. Lack of Inference Ability: Machine reading comprehension (MRC) systems retrieve the answers based on semantic matching between the question and given data. Based on the literature cited, the MRC system lacks inference ability. Research says that five persons on board and two persons on the ground have died in the given context. But the MRC system cannot retrieve the answer that is seven. This study proves that the MRC system could not infer the solution due to the lack of inference ability. This issue is still under observation ${ }^{[34]}$.

6.5. Difficulty in Interpretation: Along with many applications, the MRC system is still working in a black box, i.e., no information is given that tells how this system retrieves the answer. Lack of interpretability is its primary drawback that drops its efficiency. However, MRC models are introduced to enhance MRC systems' interpretability, such as HotpotQA ${ }^{[35]}$.

\section{FUTURE DEVELOPMENT}

India, Pakistan, and Saudi Arabia have a substantial amount of population. Their people are not well versed in English and like to communicate and learn in their language. They need to find the right information that is not possible without having an MRC system for such purposes. The languages spoken in these countries are considered low resource languages in Natural Language Processing tools development. However, a minimal amount of organized data is available in low resource language. 
This is why many MRC models have not trained adequately in low resource language due to data insufficiency.

Moving forward, we investigate and develop a plan to solve the issues that hinder the practical application of machine reading comprehension (MRC). The development, evaluation, and effectiveness of MRC systems is a crucial task. Thus this study aims to analyze what has been done in Urdu, Arabic, and Hindi language and raise research questions to develop robust MRC models that can communicate with humans using natural language.

\section{CONCLUSION}

In this paper, we present a comprehensive survey on the progress of Machine Reading Comprehension (MRC) in low resource languages in recent years. In section 1, we briefly introduced the history of MRC tasks and some early MRC systems. Moreover, section 2 sheds light on the importance of low resource languages. We demonstrate challenges in MRC Systems in section 3. Section 4 provides limitations about MRC tasks and a summary of some low resource languages dataset used to develop MRC systems in low resource languages. Furthermore, sections 5, 6, and 7 describe a thorough analysis of recent work, state-of-the-art, and recent advancement of MRC systems in low resource languages in machine learning, deep learning, and transfer knowledge. Likewise, section 8 describes some benefits of MRC, and section 9 sheds light on some new trends and discusses open issues in this research field.

Altogether, we reviewed the significant progress that has been made in recent years in the MRC field, especially in low resource languages such as Urdu, Arabic, and Hindi. However, the MRC direction is developing very fast for high resource languages, and it is difficult to utilize all newly proposed MRC work on low resource languages. We expect this survey to be a significant source to study MRC systems in low resource languages and receive worldwide researchers' attention to developing the low resource language MRC systems.

\section{ACKNOWLEDGEMENT}

The author expresses his genuine appreciation to the Deanship of Scientific Research at King Saud University, and the Research Center at the College of Languages \& Translation for their candid support.

\section{REFERENCES}

1. Q.Li, R. M. Morris, A.Fourney, K.Larson and K.Reinecke: The impact of web browser reader views on reading speed and user experience. In Proceedings of the $2019 \mathrm{CHI}$ Conference on Human Factors in Computing Systems, 2019, 1-12.

2. H.C.Lee, Y.H.Lee, L.S.Wu, L.C.Liu, W.Fang, Y.J.Hsu and H.B.Tseng. Machine comprehension of spoken content: Toefl listening test and spoken squad. IEEE/ACM Transactions on Audio, Speech, and Language Processing, 2019, 27(9), 1469-1480.

3. B.Thapa-Chhetry and T.Keck: A chrome app for improving reading comprehension of health information online for individuals with low health literacy. In IEEE/ACM 1st International Workshop on Software Engineering for Healthcare, IEEE press, 2019, 57-64.

4. S. Liu, X. Zhang, S. Zhang, H. Wang and W. Zhang. Neural machine reading comprehension: Methods and trends. Applied Sciences, 2019, 9(18), 3698. 
5. T. Dingler, B. Tag, S. Lehrer and A. Schmidt. Reading scheduler: proactive recommendations to help users cope with their daily reading volume. In Proceedings of the 17th International Conference on Mobile and Ubiquitous Multimedia, 2018, 239-244.

6. M. Amjad, G. Sidorov, A. Zhila, H. Gómez-Adorno, I. Voronkov, and A. Gelbukh. "Bend the truth": Benchmark dataset for fake news detection in urdu language and its evaluation. Journal of Intelligent \& Fuzzy Systems Preprint, 2020, 1-13.

7. R. Sharma, S. Morwal, and B. Agarwal. Named entity recognition for Hindi language: A survey. Journal of Discrete Mathematical Sciences and Cryptography, 2019, 22(4), 569-580.

8. A. Mahmood: Arabic speaker recognition system based on phoneme fusion. Multimedia Tools and Applications, 2020, 1-18.

9. E. Winter, S. Forshaw, and A. M. Ferrario: Measuring human values in software engineering. In Proceedings of the 12th ACM/IEEE International Symposium on Empirical Software Engineering and Measurement, 2018, 1-4.

10. B. Dhingra, H. Liu, Z. Yang, W. W. Cohen, and R. Salakhutdinov. Gated-attention readers for text comprehension. arXiv preprint arXiv:1606.01549, 2016.

11. M. K. Hermann, T. Kočiský, E. Grefenstette, L. Espeholt, W. Kay, M. Suleyman, and P. Blunsom,. Teaching machines to read and comprehend. arXiv preprint arXiv:1506.03340, 2015.

12. Y. Cui, W. Che, T. Liu, B. Qin, S. Wang, and G.Hu. Cross-lingual machine reading comprehension. arXiv preprint arXiv:1909.00361, 2019.

13. Y. Cui, Z. Chen, S. Wei, S. Wang, T. Liu, and G.Hu. Attention-over-attention neural networks for reading comprehension. arXiv preprint arXiv:1607.04423, 2016.

14. J. Devlin, W. M. Chang, K. Lee, and K. Toutanova. Bert: Pre-training of deep bidirectional transformers for language understanding. arXiv preprint arXiv:1810.04805, 2018.

15. N. Ferro, N. Fuhr, G. Grefenstette, A. J. Konstan, P. Castells, M. E. Daly, ... and J. Zobel. From evaluating to forecasting performance: how to turn information retrieval, natural language processing and recommender systems into predictive sciences. Dagstuhl manifestos, 2018.

16. O. Trigui, L. Hadrich Belguith, P. Rosso, H. Ben Amor, and B. Gafsaoui. Arabic QA4MRE at CLEF: Arabic question answering for machine reading evaluation, 2012.

17. H. Abdelnasser, M. Ragab, R. Mohamed, A. Mohamed, B. Farouk, M. N. El-Makky, and M. Torki. Al-Bayan: an Arabic question answering system for the Holy Quran. In Proceedings of the EMNLP Workshop on Arabic Natural Language Processing (ANLP), 2014, 57-64.

18. W. Bakari, O. Trigui, and M. Neji: Logic-based approach for improving Arabic question answering. In IEEE International Conference on Computational Intelligence and Computing Research, IEEE press, 2014, 1-6.

19. E. Al-Shawakfa: A rule-based approach to understand questions in Arabic question answering. Jordanian Journal of Computers and Information Technology (JJCIT), 2016, 2(3), 210-231. 
20. D. Gupta, S. Kumari, A. Ekbal, and P. Bhattacharyya. MMQA: A multi-domain multi-lingual question-answering framework for English and Hindi. In Proceedings of the Eleventh International Conference on Language Resources and Evaluation (LREC), 2018.

21. M. Amjad, G. Sidorov, A. Zhila. Data augmentation using machine translation for fake news detection in the Urdu language. In Proceedings of The 12th Language Resources and Evaluation Conference, 2020, 2537-2542.

22. D. Gupta, A. Ekbal, and P. Bhattacharyya: A deep neural network framework for english hindi question answering. ACM Transactions on Asian and Low-Resource Language Information Processing (TALLIP), 2019, 19(2), 1-22.

23. H.Masroor, M. Saeed, M.Feroz, K. Ahsan, K. Islam, Transtech: development of a novel translator for Roman Urdu to English. Heliyon. 2019 May 1;5(5):e01780

24. S. Romeo, G. Da San Martino, Y. Belinkov, A. Barrón-Cedeño, M. Eldesouki, K. Darwish, H. Mubarak, J. Glass, A. Moschitti, Language processing and learning models for community question-answering in Arabic. Information Processing \& Management. 2019 Mar 1;56(2):274-90

25. D. Gupta, A. Ekbal, P. Bhattacharyya. A Deep Neural Network Framework for English Hindi Question Answering. ACM Transactions on Asian and Low-Resource Language Information Processing (TALLIP). 2019 Nov 21;19(2):1-22

26. S. Viswanathan, M.A. Kumar, K.P. Soman, A Sequence-Based Machine Comprehension Modeling Using LSTM and GRU. InEmerging Research in Electronics, Computer Science and Technology 2019 (pp. 47-55). Springer, Singapore.

27. S. Gupta, and N. Khade. BERT Based Multilingual Machine Comprehension in English and Hindi. arXiv preprint arXiv:2006.01432, 2020.

28. H. Mozannar, K.E. Hajal, E. Maamary, H. Hajj, Neural Arabic question answering. arXiv preprint arXiv:1906.05394. 2019 Jun 12

29. P. Lewis, B. Oğuz, R. Rinott, S. Riedel, H. Schwenk, MLQA: Evaluating cross-lingual extractive question answering. arXiv preprintarXiv:1910.07475. 2019 Oct 16

30. D.Gupta, S. Kumari, A. Ekbal, P. Bhattacharyya, MMQA: A multi-domain multi-lingual question-answering framework for English and Hindi. InProceedings of the Eleventh International Conference on Language Resources and Evaluation (LREC 2018) 2018 May.

31. S. Liu, X. Zhang, S. Zhang, H. Wang, and W. Zhang. Neural machine reading comprehension: Methods and trends. Applied Sciences, 2019, 9(18), 3698.

32. E. Riloff, D. Chiang, J. Hockenmaier, and I. J. Tsujii: Proceedings of the Conference on Empirical Methods in Natural Language Processing. In Proceedings of the Conference on Empirical Methods in Natural Language Processing, 2018.

33. M.Amajd, Z.Kaimuldenov and I.Voronkov. Text classification with deep neural networks. In International Conference on Actual Problems of System and Software Engineering (APSSE), 2017, 364-370. 
34. K. Nishida, I. Saito, A. Otsuka, H. Asano, and J. Tomita 2018. Retrieve-and-read: Multi-task learning of information retrieval and reading comprehension. In Proceedings of the 27th ACM International Conference on Information and Knowledge Management,Press, 2018, 647-656.

35. P. P. Sinha, R. Mishra, R. Sawhney, D. Mahata, R. R. Shah and H. Liu. \# suicidal-A multipronged approach to identify and explore suicidal ideation in twitter. In Proceedings of the 28th ACM International Conference on Information and Knowledge Management, Press, 2019, 941-950.

36. X. Zhang, A. Yang, S. Li, and Y. Wang. Machine reading comprehension: a literature review. ArXiv preprint arXiv:1907.01686, 2019.

37. C. Cieri, M. Maxwell, S. Strassel, and J. Tracey: Selection criteria for low resource language programs. In Proceedings of the Tenth International Conference on Language Resources and Evaluation (LREC'16), 2016, 4543-4549.

* Corresponding Author: Dr. Mubarak Alkhatnai,

Department of English Language and Translation, College of Languages and Translation King Saud University, Riyadh, Saudi Arabia

Online date of publication: 16.04.2021 